\title{
Efficient Positioning of Data Aggregation Point for Wireless Sensor Network
}

\author{
Sifat Rahman Ahona \\ Computer Science and Engineering \\ American International University- \\ Bangladesh \\ Dhaka, Bangladesh
}

\author{
Faria Nawshin \\ Computer Science and Engineering \\ American International University- \\ Bangladesh \\ Dhaka, Bangladesh
}

\author{
Naima Hassan \\ Computer Science and Engineering \\ East West University \\ Dhaka, Bangladesh
}

\begin{abstract}
Data aggregation is a helpful technique for reducing the communication overhead in wireless sensor network. One of the important tasks of data aggregation is positioning of the aggregator points. There are a lot of work done on data aggregation. But efficient positioning of the aggregators points is not focused so much. The paper focuses on the positioning or the placement of the aggregation points in wireless sensor network. This research proposes an algorithm to select the aggregators' positions for a scenario where aggregator nodes are more powerful than sensor nodes.
\end{abstract}

\section{Keywords}

Data Aggregation, Wireless sensor network, Aggregator point, Aggregator position

\section{INTRODUCTION}

Wireless sensor network consists of different sensor node which can communicate within a small range. Sensor nodes are power constraint and have low communication range [1]. So, reducing the communication overhead is important for wireless sensor network. Data aggregation is a technique of reducing the communication in wireless sensor network. In wireless sensor network, sensors collect data and after collecting data from all nodes, data are sent to base station. Base station then processes data to generate desired output. But the problem is sometime nodes send duplicate data to the base station. Another problem is wireless sensor has very limited energy. So, whenever it sends some data to the base station it depletes some energy. So instead of sending all data to the base station data can be aggregated in some aggregator node and after that only aggregated data is sent to the base station. So which node should be the in-charge of aggregation that is important? The authors want to work on an application where nodes are scattered in a sensor field. Here authors will place some aggregator node which will be more powerful than other node. That means those aggregator nodes have more range and lifetime. This paper proposed an algorithm by modifying $\mathrm{k}$ center approximation algorithm by which one can find the minimum number of aggregator nodes which can cover the whole sensor network field.

Devices with computational, sensing and wireless communication capabilities are accumulated in Wireless sensor networks. Wireless sensor network consists of a base station along with different nodes. These nodes communicate with each other within a range. If any node wants to communicate with a distant node it uses another node as a hop. Sensor nodes sense data from different nodes and send this to the base station. These sensor nodes have a very limited lifetime. All sensors are usually scattered in an area. Sensor node sense data and forward to the base station.
Whenever data is processed some energy is consumed. When any nodes get data, it needs to forward to the base station thus more energy is lost. As sensor is very low power device energy need to be preserved. To preserve energy data aggregation is a good method. Energy consumption of the sensor networks can be minimized by determining the degree of coverage of an area and then finding out the lowest degree of coverage considering same and different sensing range of a sensor [2]. Author in [3] discusses coverage issue in detail by classification of research work with its three type of area, point and barrier coverage.

Generally, all data from different sensor nodes are sent to the base station. After that base station combine all those data to generate desired output. So, if this combining data can be done in other node before forwarding to the base station then number of forwarding would be significantly less. Thus, fairly large amount of energy is preserved. Combining data before sending to the base station in sensor node is known as data aggregation. Data aggregation reduces energy drain of sensor node as number of packets to be forwarded is reduced [4]. So, Data aggregation can be defined as a technique how data will be forwarded to the base station so that minimum energy is consumed. Bhaskar Krishnamachari defined data aggregation as Data aggregation is the task of merging messages while they are traveling through the sensor network [5]. The rest of the section is organized as follows: section 2 presents the brief related work. Section 3 describes the various techniques of data aggregations. Section 4 provides the application scenario and also describes the proposed algorithm. In the last section that is in section 5 the paper closes with a summary of the research.

\section{RELATED WORK}

Finding right aggregation point is important. There is some work done on data aggregation in wireless sensor network. Roedig gave an algorithm to calculate aggregation point and message delay [6]. In this paper they have worked with a single base station.

Some researchers have worked on structure free data aggregation. Kai-Wei Fan worked on a system where any structure maintenance is not needed. They have simulated the impact of structure free data aggregation in their paper [7].

In wireless sensor network data, which are collected from neighbor node may be redundant sometime. It may also happen that after forwarding data to the base station it may be observed that there are redundant data. There are two ways to remove data redundancy - application level and packet level. [6].

Application level aggregation: According to this approach 
data is pre-processed at the sensor node first. For example, if ten value from ten different nodes need to be sent to the base station to get the summation of those values then this summation can be done in one node of that network and only the summation result will be sent instead of those ten data.

Packet level aggregation: In this approach if any packet is seen in a network before, then that packet is dropped. For this approach compression technique or merge technique is applied to cut down overhead.

\section{DATA AGGREGATION TECHNIQUE IN WIRELESS SENSOR NETWORK}

Data aggregation in wireless sensor network is a process of sensing data from different sensor node and forwarding it to a base station. As sensors are low power device it is wise to use any aggregation technique to reduce power consumption. In large networks usually, there are huge amount of data generated and needed to forward it to the base station. So, some methods needed to combine data so that packet transmission is reduced. Data aggregation in WSN can be divided into two basic type - structure based and structure free [8][9][10][11]

\subsection{Structure Based}

This has four subcategories. Flat network, cluster-based network, tree-based aggregation and grid-based aggregation.

\subsubsection{Flat Network}

In this type of network every sensor node has same energy level. Here sink usually transmits a query message and the sensors having same query data gives a response message back to the sink. There are different types of protocol for this communication. Some of the protocols are described below:

Directed diffusion: Directed diffusion is a renowned data aggregation protocol for flat network [12]. It is a data centric approach where the data generated by nodes are described with name value pair. Thus, data coming from different source node are sent to the sink by removing redundancy. By this way it reduces transmission and save energy.

Rumor routing: Another type of directed diffusion is rumor routing [13]. It is employed where geographic routing is not supported [8]. Usually in directed diffusion query is sent to the whole network. Some cases where a little data is requested this approach is unnecessary. Here key is instead of sending query to all nodes send query to a particular node which has discovered a specific incident. This algorithm has agent which is nothing but a durable packet. Agent travels in the network to send information to distant node.

Gradient based routing: It is another type of directed diffusion where load is distributed evenly in the network to increase lifespan of the network. Here main idea is to remember the hop of that network. Thus, it is able to calculate the minimum distance to reach base station.

\subsubsection{Cluster Based Network}

This approach is applicable for large network. Here whole network is divided into different cluster. All cluster has a cluster head. Whenever data is generated in any node and needs to send to the base station then the cluster head aggregate all data as a sink and send the aggregated data to the

\section{PROPOSED ALGORITHM}

Set of sensor node $\mathrm{N}=\mathrm{s}_{1} \ldots . \mathrm{s}_{\mathrm{k}}$

Position of the Aggregator nodes $\mathrm{P}=$ base station. In large multi hopped network cluster head communicates with another cluster head. There are many aggregation protocols in cluster-based network. Leach: This is the most ancient cluster-based protocol. Here the idea is to divide in different round. Each round has two time zone. First time zone create cluster and second time zone sends data. Usually, second time zone is longer than the first time zone.

\subsubsection{Tree Based Aggregation}

In this approach nodes are organized as tree. Data aggregation is done in nodes inside that tree. This approach is more appropriate within a single network. Min Ding proposed an algorithm where the base station will send a message to all nodes and the node will start counting time [14].

\subsubsection{Grid Based Aggregation}

Here the whole network is divided into small grid. There is a aggregator in each grid which aggregates data of different nodes within that grid. Here every node communicates with its neighbor node. The aggregator node is not fixed. Any node can be the aggregator node [15].

\section{APPLICATION SCENARIOS}

A scenario of a sensor network field is considered. Some aggregator nodes have to be placed in this sensor field. And in this case the aggregator nodes are more powerful than sensor nodes. That's mean those aggregator nodes has more communication range and more battery power then sensor nodes. Also, aggregator nodes have a fixed coverage range. The aggregator nodes have to be placed in such a way that minimum number of aggregator nodes can cover the whole sensor network field.

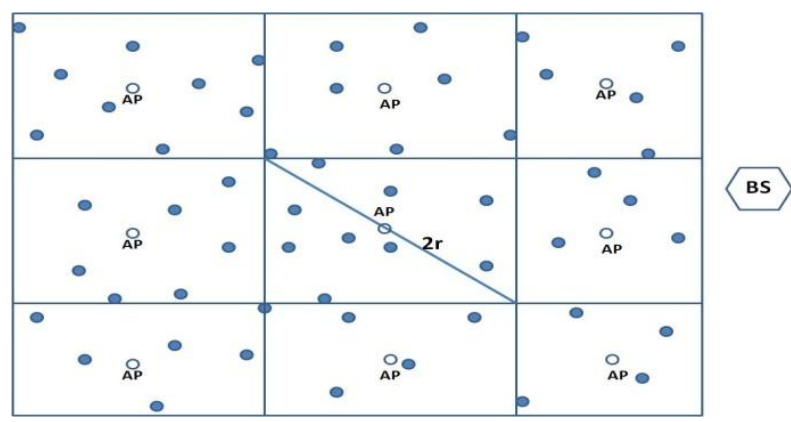

Fig 1: Sensor Field

\subsection{Selection of Aggregating Points}

Selecting the aggregation points is very crucial thing in wireless sensor network. This section discusses the method of placing the aggregation points for the aforementioned scenario. The goal is to minimize the number of aggregation nodes.

Minimum number of aggregation node is considered, which will be denoted as $\mathrm{n}$. So, all the aggregation point will be denoted as, $\mathrm{p} 1, \ldots . ., \mathrm{pn}$. Aggregator nodes have a fixed range of coverage and that will be denoted as $r$. As it is an $n p$ hard problem, a modified version of $\mathrm{k}$-center selection algorithm is proposed to select minimum number of aggregation point. The sensor field has $\mathrm{k}$ number of sensors, that means $s 1, \ldots . .$, sk. Every sensor node will be under an aggregator node, where the node will send data for aggregation. While ( $\mathrm{N}$ is not Empty)

$$
\begin{aligned}
& \mathrm{s}_{\mathrm{j}}=\text { randomly from } \mathrm{N}, \mathrm{s}_{\mathrm{j}} ; \\
& \text { Pos }=\operatorname{positionOf}\left(\mathrm{s}_{\mathrm{j}}\right) ;
\end{aligned}
$$




\author{
Add Pos in P; \\ Remove $\mathrm{s}_{\mathrm{j}}$ from $\mathrm{N}$; \\ For $\mathrm{i}=1$ to Length of $\mathrm{N}$ \\ If positionOf $\left(\mathrm{s}_{\mathrm{i}}\right)$ in $2 \mathrm{r}$ distance from Pos \\ Remove $\mathrm{s}_{\mathrm{i}}$ from $\mathrm{N}$;
}

Here $\mathrm{P}$ is the set of positions where the aggregator points can be set.

\section{RESULT ANALYSIS}

The process of collecting and aggregating the useful data is called Data Aggregation. It will save the energy that means it will save battery power and also it will cover more communication range. In WSN data aggregation is an effective way to save the limited resources.

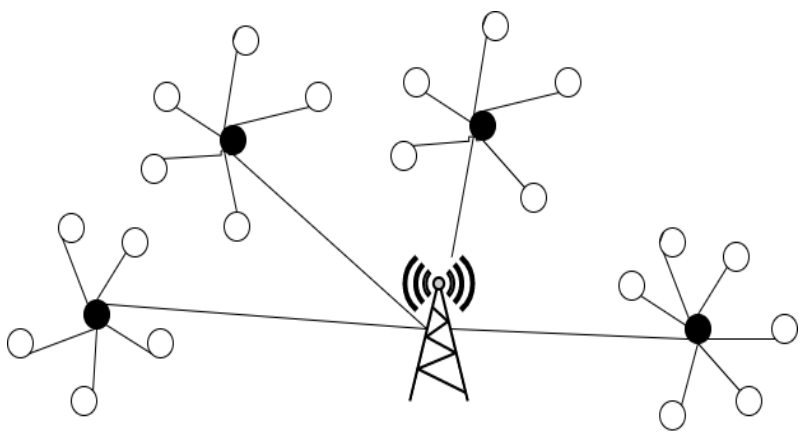

Aggregator Nodes

sensor Nodes
Base Station

Fig 2: Tested Topology

According to this algorithm, above topology in Fig 2 have tested and determined that aggregator nodes will save more battery power and cover more communication range than Sensor Nodes as shown in Fig 3. After that the number of sensor nodes have changed and aggregator nodes for the above topology considering same area coverage have also changed for further analysis.

In this topology, sensor nodes collect data and send report to the aggregator nodes which are within $2 \mathrm{r}$ distance. The aggregator nodes then send the collective report to the base station.

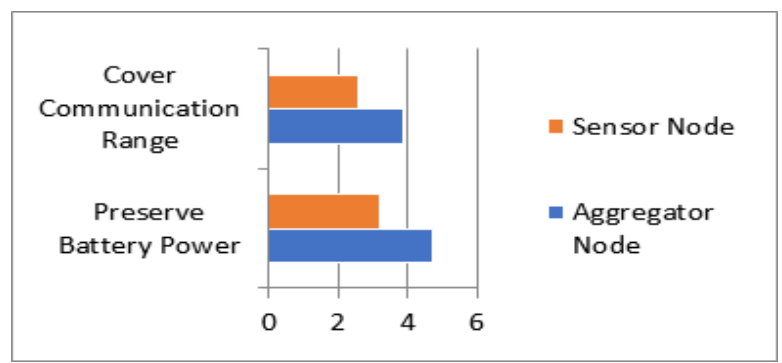

Fig 3: Comparison between Aggregator Node and Sensor Node in respect of Communication Range and Battery Power

\section{Scenario 1: Distance $=\mathbf{r}$}

Table 1. Sensor Nodes are within $r$ distance from Aggregator Nodes

\begin{tabular}{|c|c|c|c|}
\hline $\begin{array}{c}\text { Number } \\
\text { of } \\
\begin{array}{c}\text { Sensor } \\
\text { Nodes }\end{array}\end{array}$ & $\begin{array}{c}\text { Number of } \\
\text { Aggregator } \\
\text { Nodes }\end{array}$ & $\begin{array}{c}\text { Distance } \\
\text { Coverage } \\
\text { between } \\
\text { Sensor } \\
\text { Nodes and } \\
\text { Aggregator } \\
\text { Nodes }\end{array}$ & $\begin{array}{c}\text { Energy } \\
\text { Consumption } \\
\text { of Sensor } \\
\text { Nodes }\end{array}$ \\
\hline 25 & 10 & Distance $=\mathrm{r}$ & $0.29 \mathrm{~mJ}$ \\
\hline
\end{tabular}

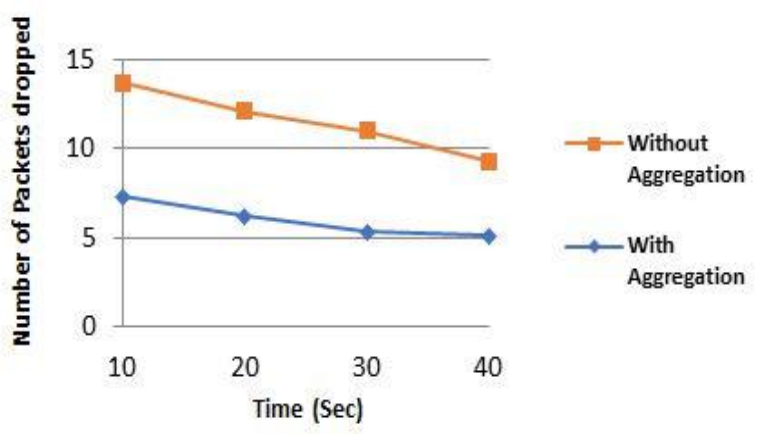

Fig 4: Comparison Between with and without Aggregation in respect of Packets Drop $($ Distance $=r$ )

Scenario 2: Distance $<\mathrm{r}$

Table 2. Sensor Nodes are less than $\mathbf{r}$ distance from Aggregator Nodes

\begin{tabular}{|c|c|c|c|}
\hline $\begin{array}{c}\text { Number } \\
\text { of } \\
\text { Sensor } \\
\text { Nodes }\end{array}$ & $\begin{array}{c}\text { Number of } \\
\text { Aggregator } \\
\text { Nodes }\end{array}$ & $\begin{array}{c}\text { Distance } \\
\text { Coverage } \\
\text { between } \\
\text { Sensor Nodes } \\
\text { and } \\
\text { Aggregator } \\
\text { Nodes }\end{array}$ & $\begin{array}{c}\text { Energy } \\
\text { Consumption } \\
\text { of Sensor } \\
\text { Nodes }\end{array}$ \\
\hline 35 & 18 & Distance $<\mathrm{r}$ & $0.38 \mathrm{~mJ}$ \\
\hline
\end{tabular}

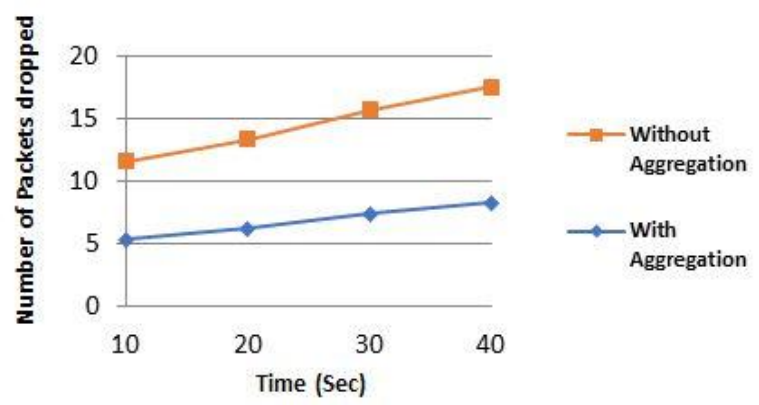

Fig 5: Comparison Between with and without Aggregation in respect of Packets Drop (Distance $<$ r) 


\section{Scenario 3: Distance $=2 \mathrm{r}$}

Table 3. Sensor Nodes are 2r distance from Aggregator Nodes

\begin{tabular}{|c|c|c|c|}
\hline $\begin{array}{c}\text { Number } \\
\text { of } \\
\text { Sensor } \\
\text { Nodes }\end{array}$ & $\begin{array}{c}\text { Number of } \\
\text { Aggregator } \\
\text { Nodes }\end{array}$ & $\begin{array}{c}\text { Distance } \\
\text { Coverage } \\
\text { between } \\
\text { Sensor } \\
\text { Nodes and } \\
\text { Aggregator } \\
\text { Nodes }\end{array}$ & $\begin{array}{c}\text { Energy } \\
\text { Consumption } \\
\text { of Sensor } \\
\text { Nodes }\end{array}$ \\
\hline 17 & 5 & $\begin{array}{c}\text { Distance } \\
2 \mathrm{r}\end{array}$ & $0.24 \mathrm{~mJ}$ \\
\hline
\end{tabular}

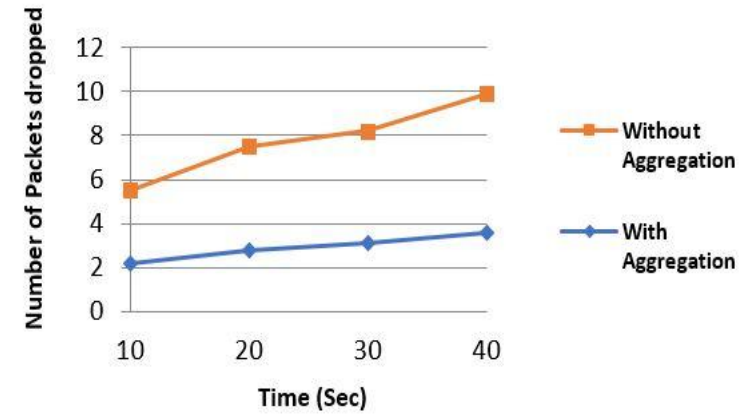

Fig 6: Comparison Between with and without Aggregation in respect of Packets Drop (Distance $=2$ r)

In this topology, aggregator nodes have been elected from sets of sensor nodes in such a way that all other sensor nodes are within $2 \mathrm{r}$ distance from the elected aggregator nodes and all the aggregator nodes have been connected to a base station. According to this projected algorithm, number of packets drop is very less with the application of the data aggregation algorithm shown in Fig 9.

The proposed algorithm for three different scenarios has tested and obtained following comparative result in Fig 10 by changing few parameters like distance between sensor nodes and aggregator nodes, number of sensor nodes, aggregator nodes with same area coverage.

Table 4. Comparison among Different Parameters when considering three distances

\begin{tabular}{|c|c|c|c|c|}
\hline $\begin{array}{c}\text { Distance } \\
\text { from } \\
\text { sensor } \\
\text { nodes to } \\
\text { aggregator } \\
\text { nodes }\end{array}$ & $\begin{array}{c}\text { Require } \\
\text { Sensor } \\
\text { Nodes }\end{array}$ & $\begin{array}{c}\text { Require } \\
\text { Aggregator } \\
\text { Nodes }\end{array}$ & $\begin{array}{c}\text { Power } \\
\text { Consu } \\
\text { mption }\end{array}$ & $\begin{array}{c}\text { Packet } \\
\text { Drop }\end{array}$ \\
\hline $\mathrm{r}$ & More & Less & High & High \\
\hline$<\mathrm{r}$ & More & More & High & High \\
\hline $2 \mathrm{r}$ & Less & Less & Less & Less \\
\hline
\end{tabular}

\section{CONCLUSION AND FUTURE WORK}

In this work an aggregation point selection algorithm is proposed. The algorithm can select the minimum number of aggregators point for a sensor network field. This is a greedy approximation algorithm. The described method of selecting aggregator node of this paper will only work in a scenario where aggregator nodes are more powerful than sensor nodes, they have a capability of a single hop communication with base station. And this is applicable for the scenario where battery lifetime of the sensor node is not in the consideration that's mean sensor nodes are deployed for a small amount of time for sensing some phenomena.

Here, the authors are working on a fixed topology. In the future upgradation of this algorithm authors will try to incorporate the idea of frequently changing topology. Authors are also planning to simulate the algorithm and analyze the result on a real scenario.

\section{REFERENCES}

[1] J. Yick, B. Mukherjee, and D. Ghosal, "Wireless sensor network survey," Computer Networks, vol. 52, no. 12, pp. 2292-2330, 2008.

[2] Patra, R. R., \& Patra, P. K. "Analysis of k-coverage in wireless sensor networks." International Journal of Advanced Computer Science and Applications, 2.9(2011)

[3] Singh, Abhiram, and T. P. Sharma. "A survey on area coverage in wireless sensor networks." 2014 International Conference on Control, Instrumentation, Communication and Computational Technologies (ICCICCT). IEEE, 2014

[4] Tarnaris, K., Preka, I., Kandris, D., \& Alexandridis, A. (2020). Coverage and k-Coverage Optimization in Wireless Sensor Networks Using Computational Intelligence Methods: A Comparative Study. Electronics, 9(4), 675 .

[5] B. Krishnamachari, D. Estrin, and S. B. Wicker, "The Impact of Data Aggregation in Wireless Sensor Networks," ICDCS Workshops, pp. 575-578, 2002.

[6] U. Roedig, A. Barroso, and C. Sreenan, "Determination of aggregation points in wireless sensor networks," Proceedings. 30th Euromicro Conference, 2004., pp. 503- 510, 2004. [Online]. Available: http://ieeexplore.ieee.org/lpdocs/epic03/wrapper.htm?arn umber $=1333413$

[7] K. W. Fan, S. Liu, and P. Sinha, "Structure-free data aggregation in sensor networks," IEEE Transactions on Mobile Computing, vol. 6, no. 8, pp. 929-942, 2007.

[8] V. Pandey, "A review on data aggregation techniques in wireless sensor network," Journal of Electronic and Electrical Engineering, vol. 1, no. 2, pp.1-8, 2010. [Online]. Available: http://www.bioinfo.in/uploadfiles/12937857621\{ \}2\{ \}1\{ \}JEEE.pdf

[9] N. Xu, A. Broad, and D. Estrin, "A Wireless Sensor Network For Structural Monitoring Categories and Subject Descriptors," 2004.

[10] C. Schurgers and M. B. Srivastava, "Energy Efficient Routing in Wireless Sensor Networks," IEEE Military Communications Conference, 2001. MILCOM 2001, vol. 1, no. c, pp. 357-361, 2001. [Online]. Available: http://ieeexplore.ieee.org/lpdocs/epic03/wrapper.htm?arn umber=985819 $\{\%\}$ Cnhttp://www.shihada.com/F12344/ papers/EnEff $\{$ \}routing \{\} wsn.pdf $\{\%\} 5 \mathrm{Cnhttp}: / /$ ieeexplor e.ieee.org/xpls/abs \{ \}all.jsp?arnumber=985819

[11] W. R. Heinzelman, A. Chandrakasan, and H. Balakrishnan, "Energy-Efficient Communication 
Protocol forWireless Microsensor Networks," Hawaii International Conference on System Sciences, 2000.

[12] B. R. Szewczyk, E. Osterweil, J. Polastre, M. Hamilton, A. Mainwaring, and D. Estrin, "Directed Diffusion for Wireless Sensor Networking," Communications of the ACM, vol. 47, no. 6, pp. 34-40, 2006. [Online]. Available: http://ieeexplore.iee.org/xpls/abs $\{\quad\}$ all.jsp?arnumber $=1180542$

[13] D. Braginsky and D. Estrin, "Rumor routing algorithm for sensor networks," Proceedings of the 1st ACM International Workshop on Wireless Sensor Networks and Applications (WSNA), pp. 22-31, 2002. [Online].
[14] M. Ding, X. Cheng, and G. Xue, "Aggregation tree construction in sensor networks," 2003 IEEE 58th Vehicular Technology Conference. VTC 2003-Fall (IEEE Cat. No.03CH37484), vol. 4, pp. 2168-2172, 2003.

[15] K. Vaidyanathan, S. Sur, S. Narravula, and P. Sinha, "Data Aggregation Techniques in Sensor Networks Karthikeyan," Network, no. OSU-CISRC-11/04-TR60, pp. 71-82, 2006. [Online]. Available: http://citeseerx.ist.psu.edu/viewdoc/download?doi=10.1. $1.84 .9503\{\&\}$ rep $=$ rep $1\{\&\}$ type $=$ pdf 